\title{
Frequency and Risk Factors of Retinopathy of Prematurity in Newborns Admitted to Neonatal Intensive Care Unit in Hamadan
}

\author{
Behnaz Basiri' ${ }^{1}$ Mohammad Kazem Sabzehei ${ }^{1}$, Maryam Shokouhi Solgi ${ }^{2}$, Siamak \\ Akbarzadeh $^{3, *}$, ID , Somaye Ivazeh ${ }^{4}$ \\ ${ }^{1}$ Associate Professor, Department of Pediatrics, School of Medicine, Hamadan University of Medical Sciences, Hamadan, \\ Iran \\ ${ }^{2}$ Assistant Professor, Department of Pediatrics, School of Medicine, Hamadan University of Medical Sciences, Hamadan, \\ Iran \\ ${ }^{3}$ Associate Professor, Department of Ophthalmology, School of Medicine, Hamadan University of Medical Sciences, \\ Hamadan, Iran \\ ${ }^{4}$ Resident in Psychiatry, School of Medicine, Tabriz University of Medical Sciences, Tabriz, Iran \\ * Corresponding Author: Siamak Akbarzadeh, Department of Ophthalmology, School of Medicine, Hamadan University of \\ Medical Sciences, Hamadan, Iran.Email: siakbarzadeh65@yahoo.com
}

Received: 13.12 .2018 Accepted: 13.04 .2019

How to Cite this Article: Basiri B, Sabzehei MK, Shokouhi Solgi M, Akbarzadeh S, Ivazeh S Frequency and Risk Factors of Retinopathy of Prematurity in Newborns Admitted to Neonatal Intensive Care Unit in Hamadan. Avicenna J Clin Med. 2019 26(1): 44-50. DOI: $10.29252 /$ ajcm.26.1.44

\section{Abstract}

Background and Objective: Retinopathy of prematurity (ROP) is an evolutionary disorder in the retina of premature infants. Nowadays, the prevalence rate of retinopathy has increased due to the advancement of medical facilities and the increased survival of preterm infants. The aim of this study was to determine the prevalence rate of ROP and its related risk factors among infants admitted to and examined at neonatal intensive care unit (NICU) affiliated to Fatemieh Hospital in Hamadan.

Materials and Methods: This descriptive cross-sectional study was conducted on all premature infants selected with census sampling methods and with a gestational age of fewer than 34 weeks who were hospitalized in NICU for four weeks or more. The premature infants were investigated regarding the incidence of retinopathy and its risk factors.

Results: Out of 80 neonates under study, 24 (30\%) cases suffered from ROP, of which 16 and 8 neonates were in stage I and II of ROP, respectively. Moreover, the mean gestational age of newborns was $29.71 \pm 2.16$ weeks, and more than $75 \%$ of ROP were observed in neonates under 29 weeks' gestational age. In addition, the results revealed that ROP correlated significantly with gestational age, low birth weight, $1^{\text {st }}$ and $5^{\text {th }}$ minute Apgar scores, the need to resuscitation, the use of inotropic drugs, and duration of oxygen therapy. However, there was a significant relationship between low birth weight and retinopathy based on the results obtained from logistic regression.

Conclusion: The prevalence rate of ROP stands on average regarding the results obtained from Iranian and foreign published data. Among the independent variables that affect retinopathy, low birth weight was the only significant variable affecting the incidence of retinopathy.

Keywords: Infant, Low Birth Weight, Premature, Retinopathy of Prematurity, Risk Factors 


\title{
فراوانى و عوامل خطر رتينوياتى نارسى در نوزادان بسترى در بخش مراقبتهاى ويزه نوزادان همدان
}

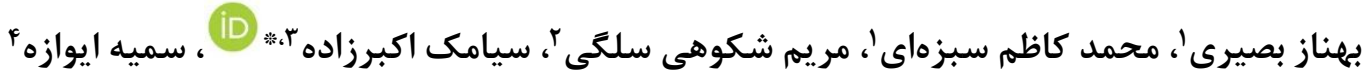

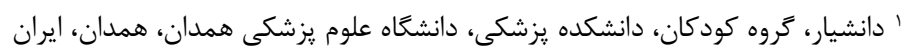

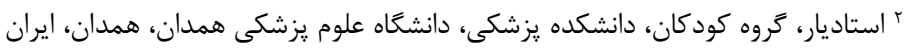

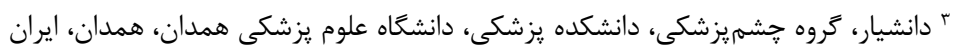

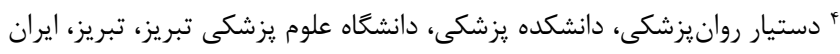

* نويسنده مسئول: سيامك اكبرزاده، كروه جشميزشكى، دانشكده يزشكى، دانشكاه علوم يزشكى همدان، همدان، ايران.

ايميل: siakbarzadeh65@yahoo.com

\begin{tabular}{|c|c|}
\hline \multicolumn{2}{|l|}{ جكيده ج } \\
\hline سابقه و هدف: رتينوياتى نارسى (ROP: Retinopathy of Prematurity) يك اختلال تكاملى در عروق & 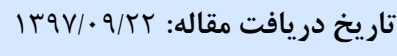 \\
\hline شبكيه هشم نوزادان نارس است. امروزه با پِيشرفت امكانات يِ شكى و افزايش بقاى نوزادان نارس، ميزان شيوع & 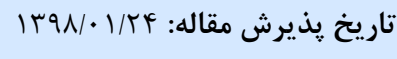 \\
\hline رتينوياتى افزايش يافته است. در اين راستا، مطالعه حاضر با هدف تعيين فراوانى رتينوياتى نارسى و عوامل & \\
\hline خطام شد. & 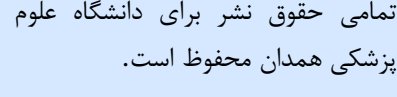 \\
\hline \multirow{2}{*}{\multicolumn{2}{|c|}{ 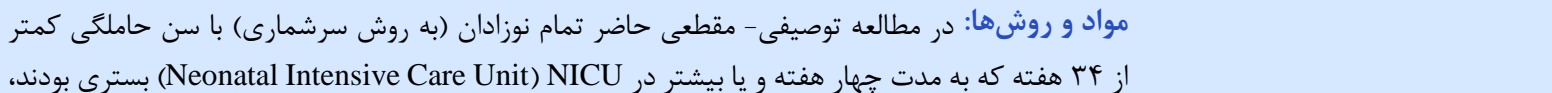 }} \\
\hline & \\
\hline \multicolumn{2}{|l|}{ از نظر بروز رتينوياتى و ريسكفاكتورهاى مؤثر در ايجاد آن مورد بررسى قرار كرفتند. } \\
\hline \multicolumn{2}{|l|}{ 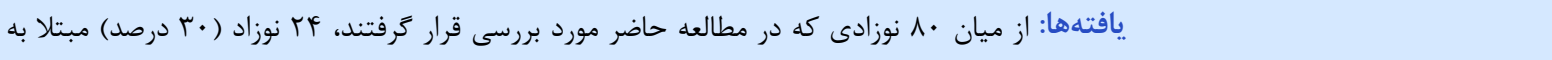 } \\
\hline \multicolumn{2}{|l|}{ رتينوياتى نارسى بودند كه از اين تعداد، 1 انوزاد در مرحله يك و هشت نوزاد در مرحله دو قرار داشتند. } \\
\hline \multicolumn{2}{|l|}{ 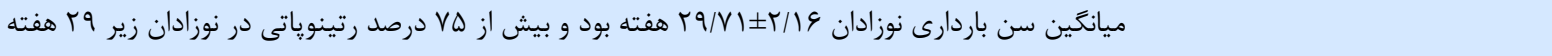 } \\
\hline \multicolumn{2}{|l|}{ مشاهده شد. اكر جه با محاسبه ارتباط متغيرهاى مستقل با رتينوياتى بهصورت جداكانه بين رتينوياتى نارسى } \\
\hline \multicolumn{2}{|l|}{ با سن باردارى، وزن هنكام تولد، نمره آيخار دقيقه اول و ينجم، نياز به احيا، استفاده از داروهاى اينوتروب و } \\
\hline \multicolumn{2}{|l|}{ مدت دريافت اكسيرن ارتباط آمارى معنادارى مشاهده شد؛ اما در محاسبه ركرسيون لجستيك تنها بين وزن } \\
\hline \multicolumn{2}{|l|}{ هنكام تولد با رتينوياتى ارتباط آمارى معنادارى وجود داشت. } \\
\hline \multicolumn{2}{|l|}{ نتيجه كَيرى: بروز رتينوياتى در نوزادان نارس متولدشده در محدوده متوسط آمارهاى داخلى و خارجى است. } \\
\hline \multicolumn{2}{|l|}{ از بين متغيرهاى مستقل اثركذار بر رتينوياتى، وزن كم هنكام تولد تنها متغير معنادار مؤثر بر بروز رتينوياتى } \\
\hline 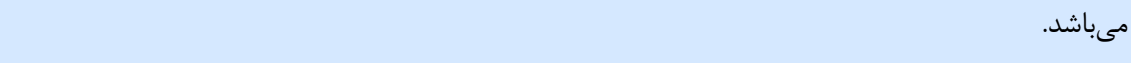 & \\
\hline وازَّان كليدى: رتينوياتى نارسى، عوامل خطر، نوزاد نارس، وزن كم نوزاد هنحام تولد & \\
\hline
\end{tabular}

مقلدمه

"نئوواسـكولاريزاسـيون" كَفته مىشــود كه منجر به بيمارى

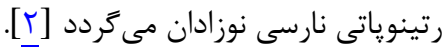

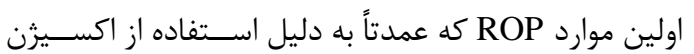

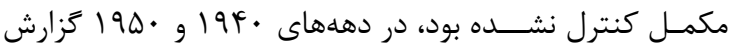

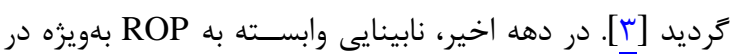

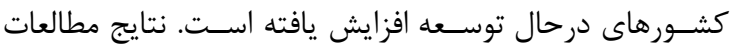

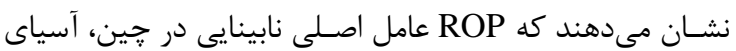

رتينوياتى نارسـى كه در كَذشـته تحت عنوان "فيبرويلازى

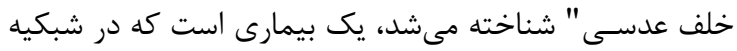

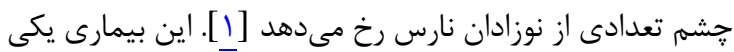

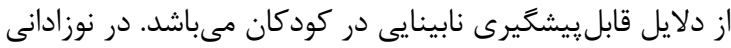

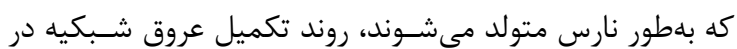

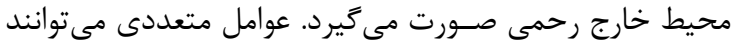

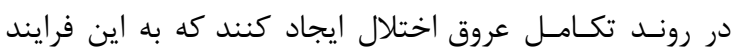


جهار هفته مرخص شـده بودند، نوزادانى كه قبل از تهار هفته

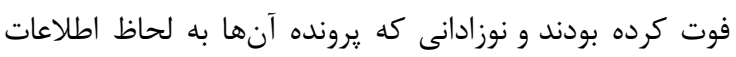

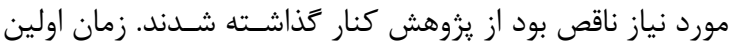

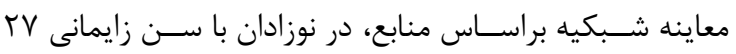

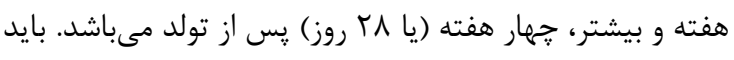

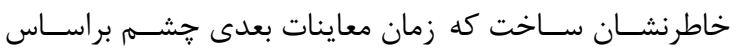

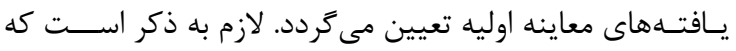

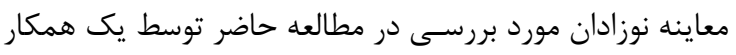

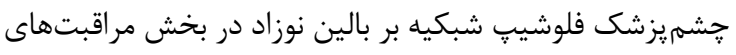

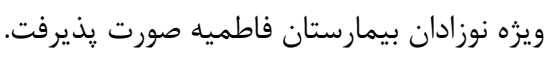

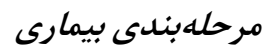

ل Zone I

برابر فاصله fovea تا مركز ديسك إيتيك است. Zone II

$$
\text { ناحيه نزديك اكواتور تميورال }
$$

Zone II ناحيه هلالى شكل در قدام :Zone III

Demarcation line وجود: Stage 1

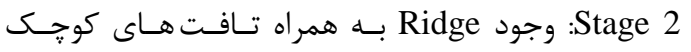

$$
\text { يروليفراسيون فيبروواسكولار }
$$

كمراه با يروليفراسـيون فيبروواسـكولار 3

$$
\text { اكسترا رتينال }
$$

فو آ؛ Stage 4

$$
\text { فووه آ؛ B: با دركيرى فووه آ }
$$

Stage 5

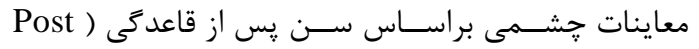

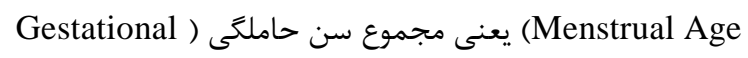

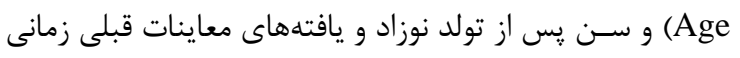

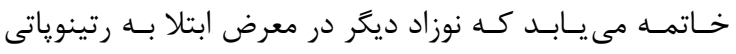

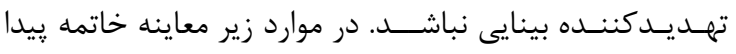
مى كند: I. تكميل رگدارشـدن طبيعى شبكيه تا انتهاى زون II كه

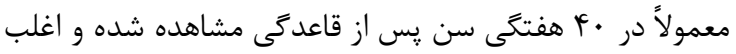

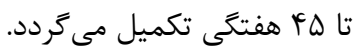

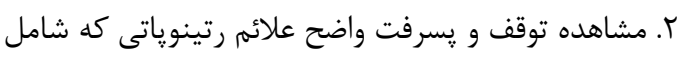
موارد زير است: الف. تغيير رنغ لبهها از صورتى به سفيد

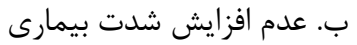

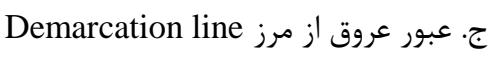
د. شروع فرايند جايكزينى ضايعات فعال با بافت اسكار

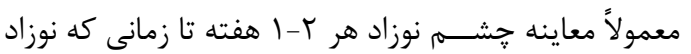

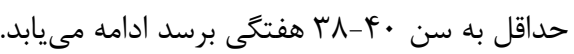

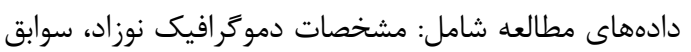

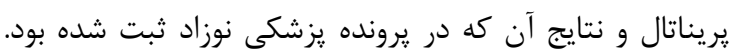

جنوب شـرقى، آمريكاى جنوبى، آمريكاى لاتين و اروياى شرقى

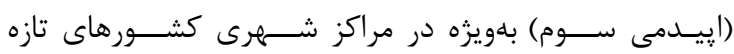

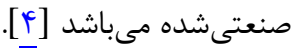

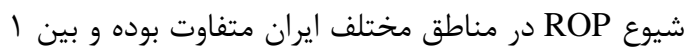

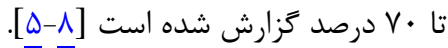

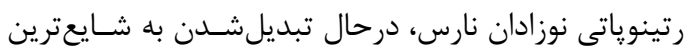

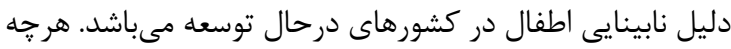

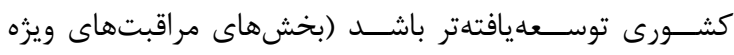

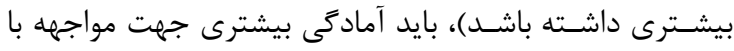

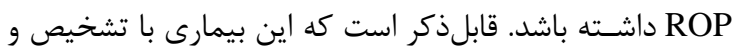

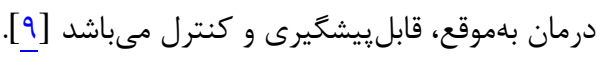

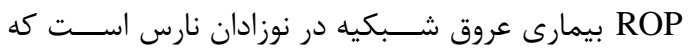

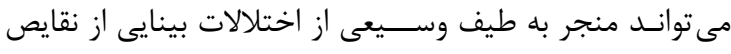

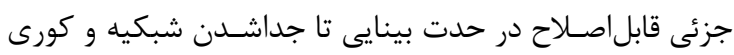

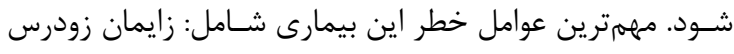

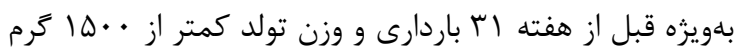

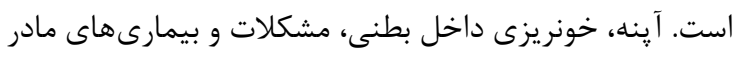

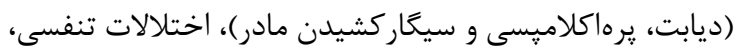

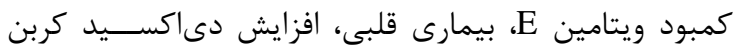

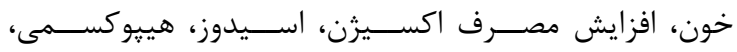

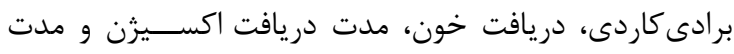

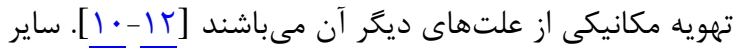

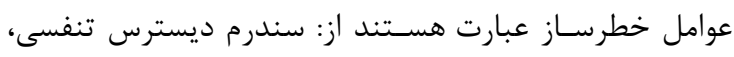

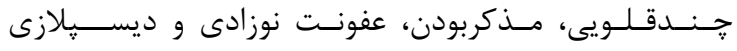

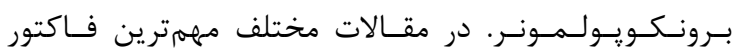

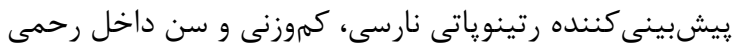
يايين ززارش شده است [11ا]. اين بيمارى در اغلب موارد قابل ييشـخيرى بوده و در صر صورت

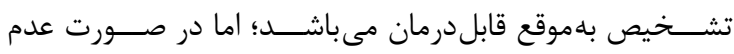

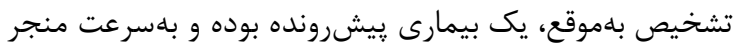
به نابينايى مى كردد. با توجه به اهميت موارد ذكرشــده و افزايش بقاى نود نوزادان

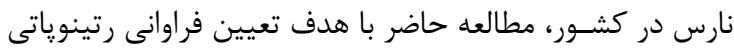

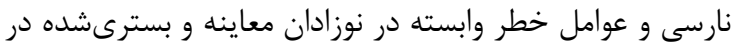
بخش مراقبتهاى ويزه بيمارستان فاطميه همدان انجام شد.

\section{مواد و روشها}

مطالعه توصيفى - مقطعى حاضر به مدت يك سال از ابتداى

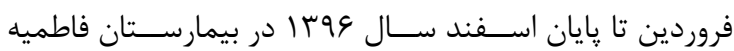
وابسـته به دانشعاه علوم يزشكى همدان انجام شد. تمام نوزادان

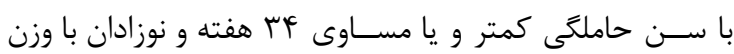

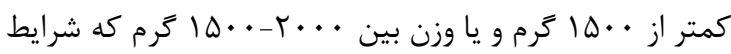

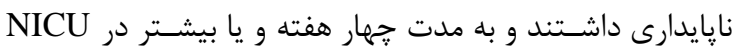

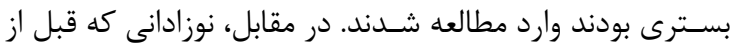


بين فراوانى رتينوياتى نارسى و سن باردارى، ارتباط آمارى

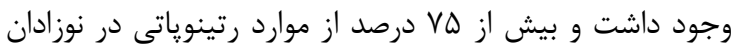
كمتر از وץ هفته رخ داده بود (جدول () ).

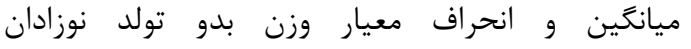
r r/r بود. ميانگين وزن نوزادان مبتلا به رتينوياتى و غيرمبتلا به آن نيز

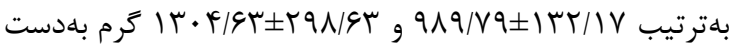

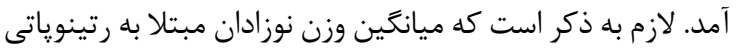

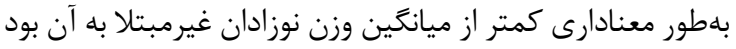
.$\left(\chi^{2}=r / 9 \uparrow ، \mathrm{df}=\mathrm{V} \wedge \mathrm{d}=\cdot / \cdot \cdot 1\right)$

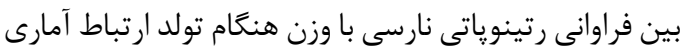

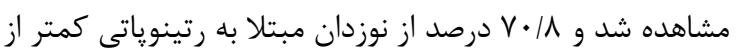

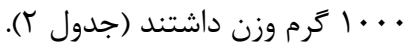

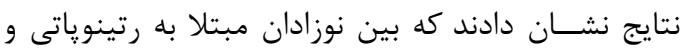

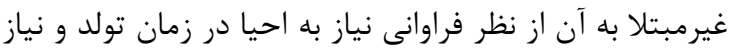

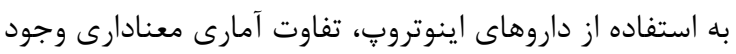

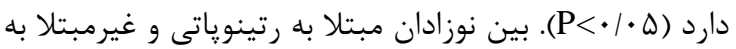

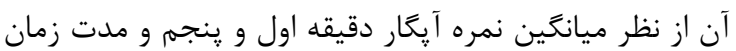

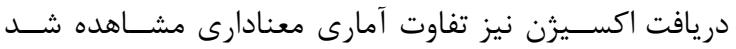

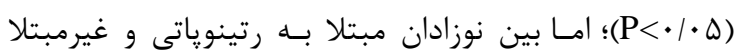

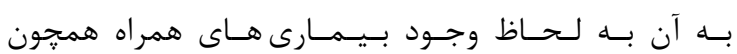
Intra ) IVH ،Respiratory Distress Syndrome) RDS (Ventricular Hemorrhage آمارى معنادارى مشاهده نخرديد (P=•/DV9).

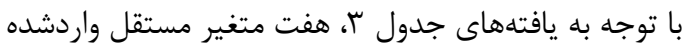

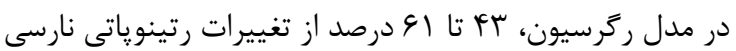
را ييشبينى نمودند. براساس نتايج از بين متغيرهاى مستقل، تنهـا

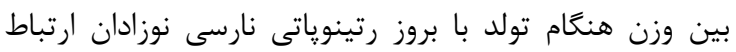

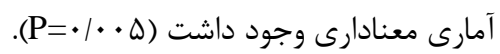

جهت انجام اين يزوهش، سوابق رتينوياتى از : يرونده يزشكى

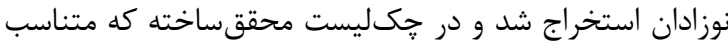
با اهداف يزوهش بود، ثبت گرديد.

SPSS 21 در مطالعه حاضر براى تحليل دادهها از نرمافئ بوديل

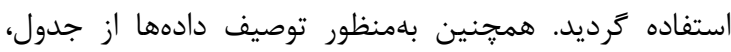
نمودار، نسبت، درصد و شاخصهاى مركزى و يراكندگى استفاده

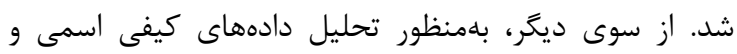

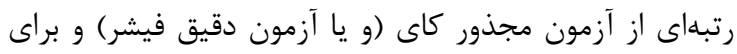

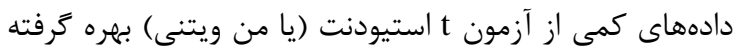

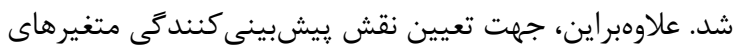

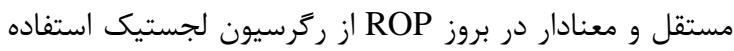

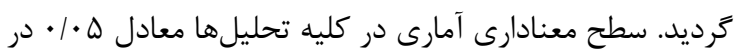
نظر گرفته شد.

\section{1.}

در مجموع براى • 1 نوزادى كه تا سن جهار هفتخى در بخش

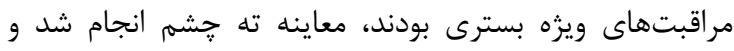

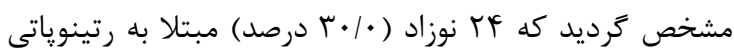

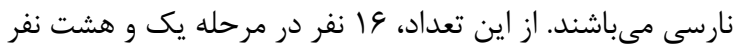

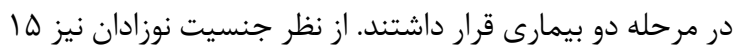

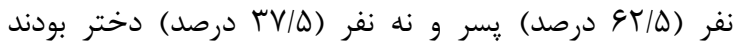
.$\left(\chi^{2}=r / 9 \cdot d \mathrm{df}=1, \mathrm{P}=\cdot / \cdot r \mathrm{~V}\right)$ در اين مطالعه ميانگين و انحراف معيار سن باردارى نوزادان.

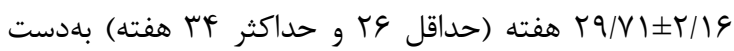

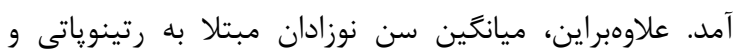

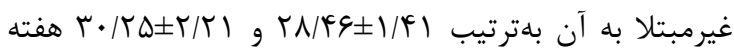

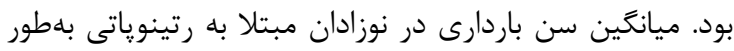

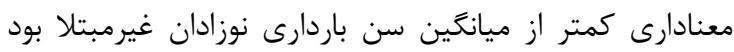
. $\left(\chi^{2}=r / 90\right.$ dff $\left.=V \wedge ، \mathrm{P}=\cdot / \cdot \cdot 1\right)$

جدول ا: فراوانى نوزادان مورد بررسى به تفكيك سن باردارى و مبتلا يا غيرمبتلابودن به رتينوياتى

\begin{tabular}{|c|c|c|c|c|}
\hline سطح معنادارى & مجموع تعداد (درصد) & بدون رتينوياتى تعداد (درصد) & داراى رتينوياتى تعداد (درصد) & سن باردارى (هفته) \\
\hline \multirow{4}{*}{$\cdot / \cdot r F$} & $(1 \cdots) r \wedge$ & $(\Delta \cdot) \mid f$ & $(\Delta \cdot) \mid f$ & r^ا r r \\
\hline & $(1 \cdots) r \Lambda$ & $(V / / \mathcal{F}) \Gamma \cdot$ & $(Y \wedge / 9) \wedge$ & r. \\
\hline & $(1 \cdots) 1$. & $(9 \cdot) 9$ & $(1 \cdot) 1$ & וr تا rr \\
\hline & $(1 \cdots) \mid f$ & $(\wedge \Delta / V) \backslash T$ & $(\mid r / T) T$ & rr تا r \\
\hline
\end{tabular}

جدول ب: فراوانى نوزادان مورد بررسى به تفكيك وزن هنگًام تولد و مبتلا يا غيرمبتلابودن به رتينوياتى

\begin{tabular}{|c|c|c|c|}
\hline سطح معنادارى & بدون ر تينوياتى تعداد (درصد) & داراى رتينوياتى تعداد (درصد) & وزن هنَام تولد (كَم) \\
\hline \multirow{4}{*}{$\begin{array}{l}<\cdot 1 \cdot \cdot 1 \\
<\cdot 1 \cdot \cdot 1 \\
<\cdot 1 \cdot \cdot 1\end{array}$} & $<\cdot / \cdot l^{*}(\mid \kappa / \mu) \wedge$ & $(V \cdot \mid \Lambda) \mid V$ & زير ...1 \\
\hline & $(G r / \Delta) r \Delta$ & $(Y q / \zeta) \vee$ & 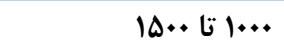 \\
\hline & (rT/T) & $(\cdot) \cdot$ & •."ها و بالاتر \\
\hline & $(1 \cdots) \Delta \varphi$ & $(1 \cdots)$ TF & مجموع \\
\hline
\end{tabular}


جدول ب: ضرايب ركرسيون لجستيك متغيرهاى مستقل بِيشبينى كننده رتينوياتى نارسى نوزادان

\begin{tabular}{|c|c|c|c|c|c|c|}
\hline $\operatorname{Exp}(B)$ & Sig. & Df & Wald & S.E. & B & \\
\hline .922 & .822 & 1 & .051 & .360 & -.081 & سن حاملكى \\
\hline .991 & .005 & 1 & 7.869 & .003 & -.009 & | وزن بدو تولد \\
\hline .300 & .084 & 1 & 2.985 & .697 & -1.204 & |آيخار دقيقه اول \\
\hline .483 & .249 & 1 & 1.330 & .631 & -.727 & |آيگًار دقيقه ينجم \\
\hline 2.802 & .497 & 1 & .462 & 1.516 & 1.030 & نياز به احيا \\
\hline 1.295 & .762 & 1 & .092 & .853 & .258 & نياز به تجويز داروهاى اينوتروڤ \\
\hline .983 & .851 & 1 & .035 & .091 & -.017 & مدت زمان دريافت اكسيزن \\
\hline 4.839E8 & .071 & 1 & 3.267 & 11.063 & 19.997 & Constant \\
\hline
\end{tabular}

مطالعه نشـان دادند كه سن حاملگى و وزن تولد در مبتلايان به

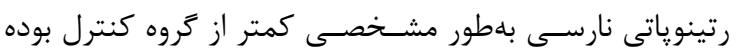

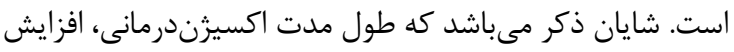

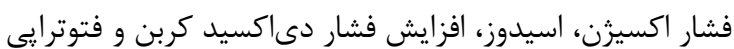

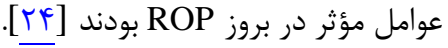

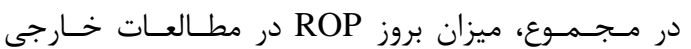

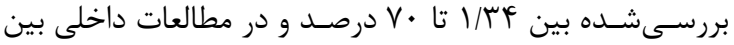

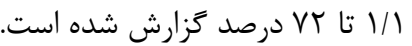

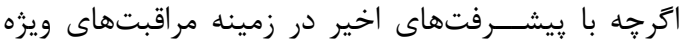

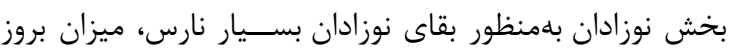

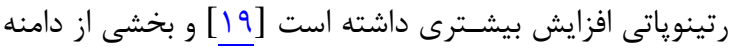

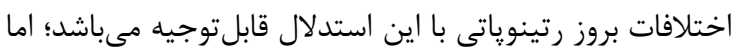

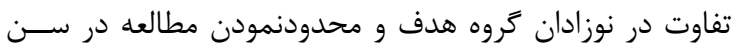

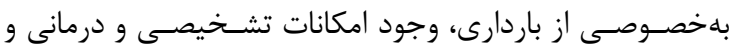
اختلاف در حجم نمونه مورد بررسى مى توانند توجيه كننده ساير

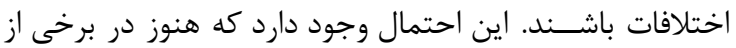
بيمارستانها بهصورت كنترلنشدهاى از مكمل اكسيرن استفاده إنداه

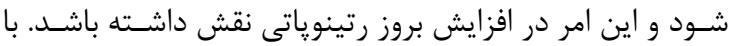

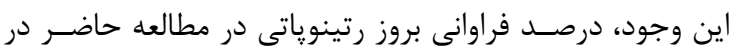
دامنه مطالعات ذكرشده قرار دارد.

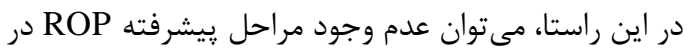

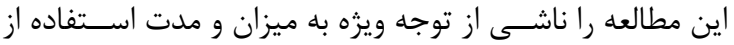
اكسـيزن مكمل در بخش مراقبتهاى ويزه بيمارسـتان فاطميه،

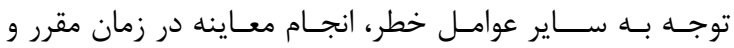

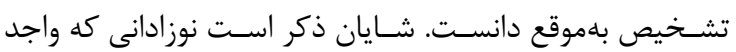

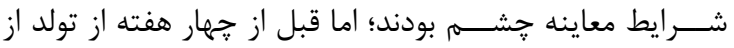

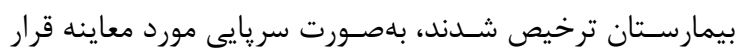
گرفتند و از مطالعه حذف گرديدند.

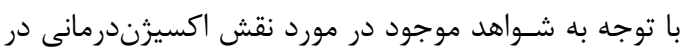

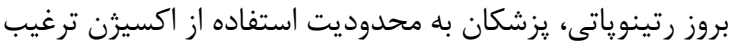

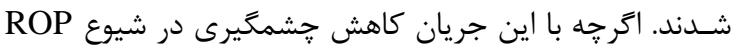

بروز رتينوياتى در مطالعه حاضـر ·r درصـد بود و بلترتيب

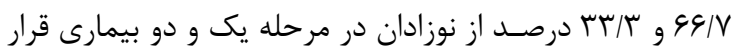

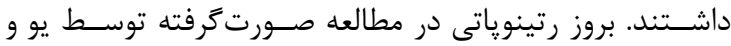

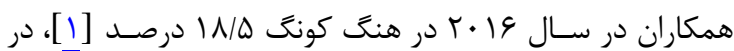

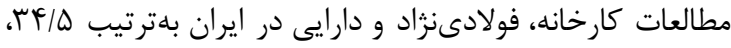

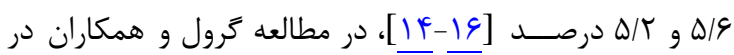

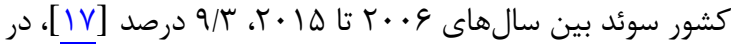

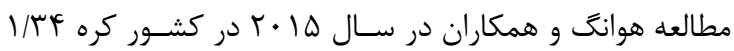

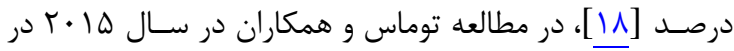

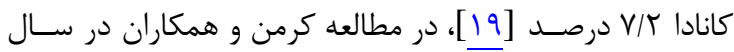

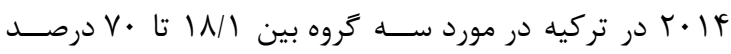

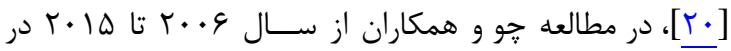

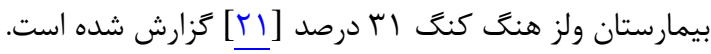

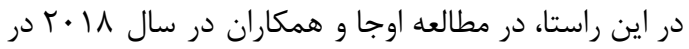

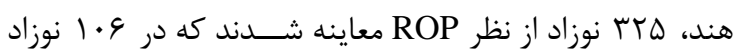

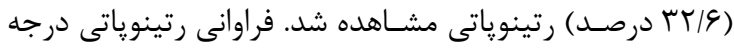

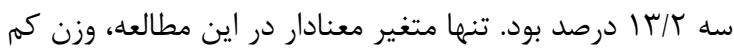

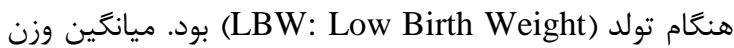

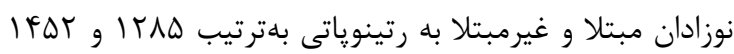

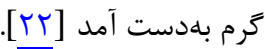
در مطالعات انجامشـده در داخل كشــور نيز بروز رتينوياتى إنى

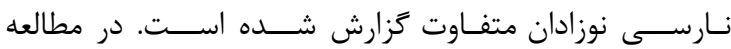

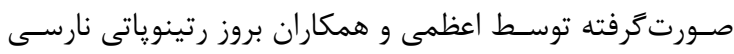

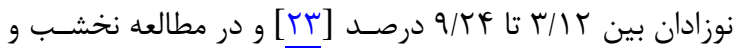

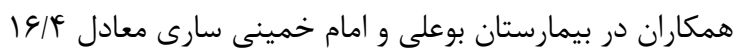
درصد [^] ثبت گرديده است.

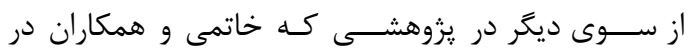

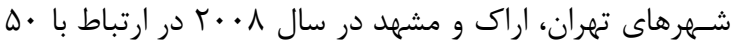

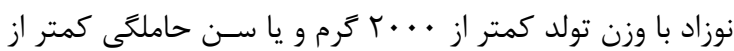

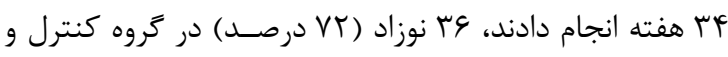

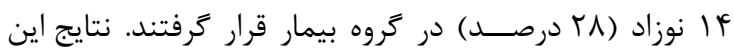


رتينوياتى نارسـى يكى بيمارى جند علتى مىباشــد، به نظر

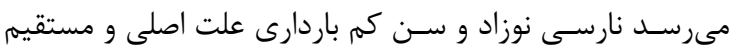

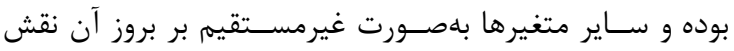

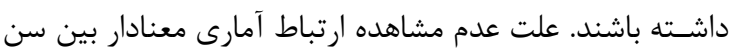

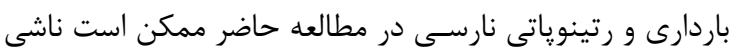

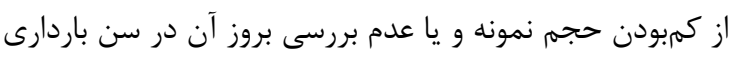

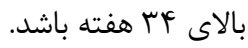
از سـوى ديخر، مطالعات متعددى ارتباط آمارى معنادار بين باتند

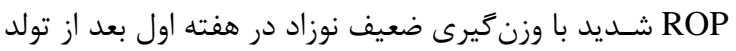

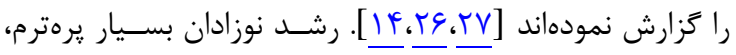

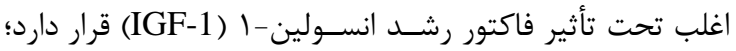

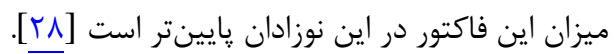

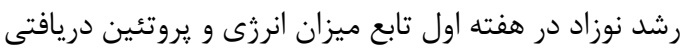

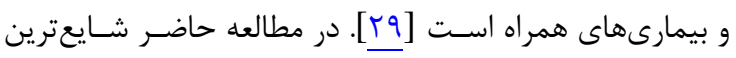

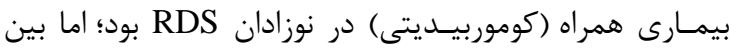

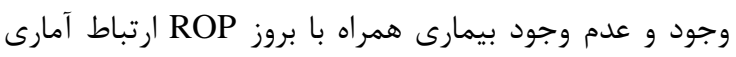

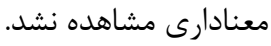
در برخى از مطالعات، تزريق خون بهعنوان يك فاكتئور

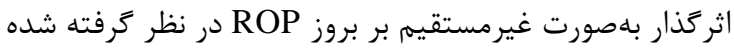

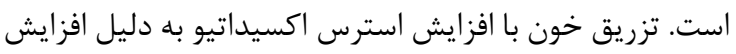

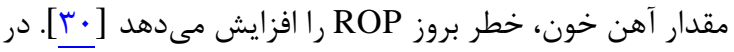
مطالعه حاضر در مجموع ب r نفر خون درئ دريافت نموده بودند

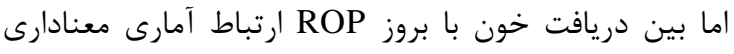

مشاهده نشد.

\section{نتيجه گيرى}

بروز رتينوياتى در نوزادان نارس كمتر از ب M هفته متولد و

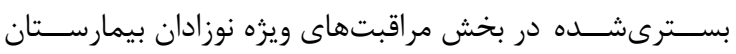

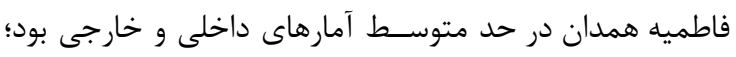

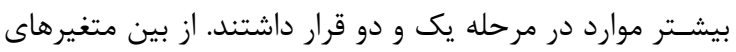

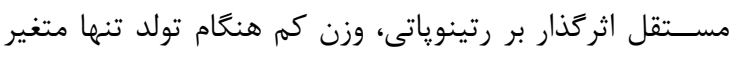

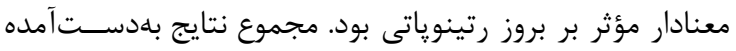

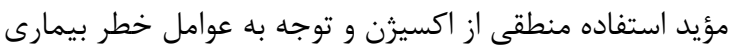
در محيط يزووهش مىباشد.

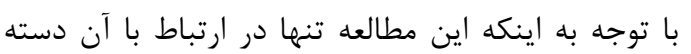

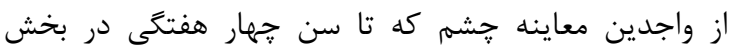

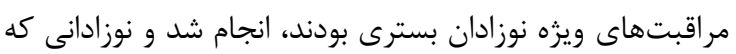

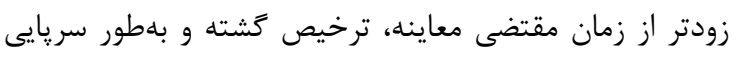

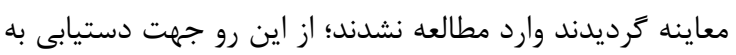

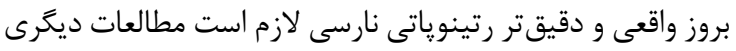
طراحى گرديده و انجام شوند.

\section{تشكر و قلر دانى}

مقاله حاضـــر بركرفته از پاياننامه دوره دكترى حرفهاى

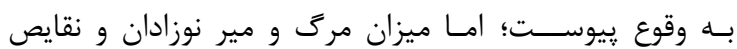
نورولوزيكى آنها همجون فلج مغزى (CP: Cerebral Palsy)

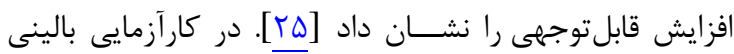

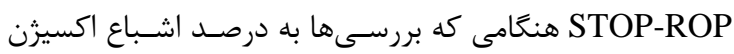
خون شـريانى و نقش اكسـيزن تكميلى معطوف گرديد، نشـان

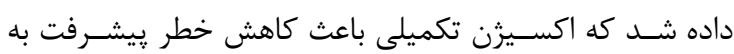

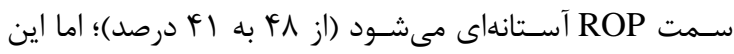

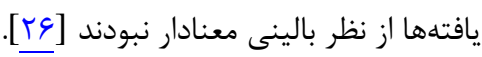

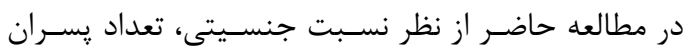

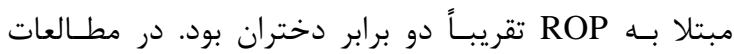

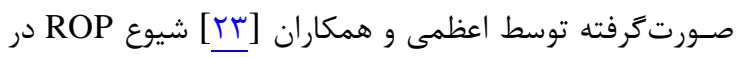

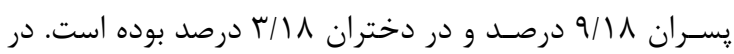

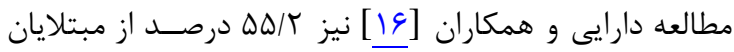

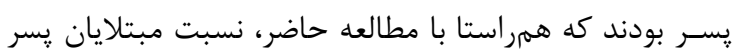
بيشتر از دختران بود.

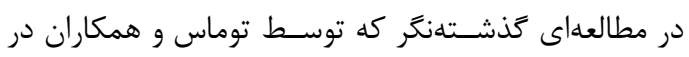

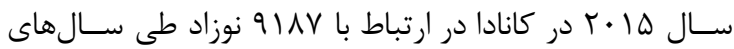

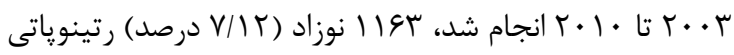

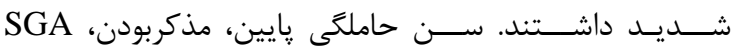

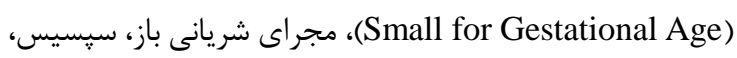

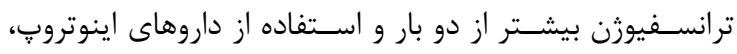

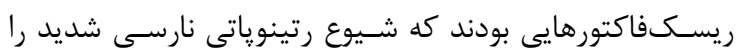

افزايش داده بودند [19].

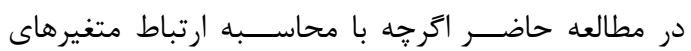

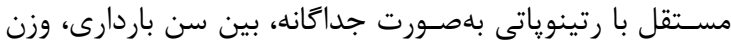

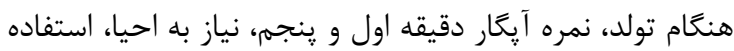

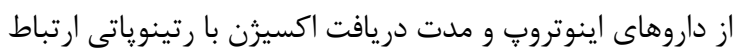

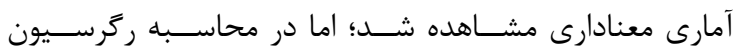

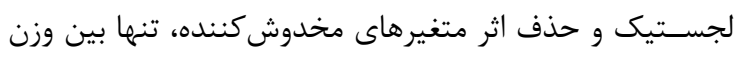

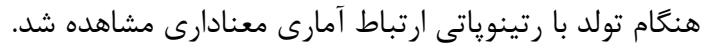

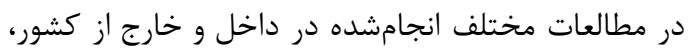

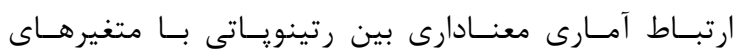

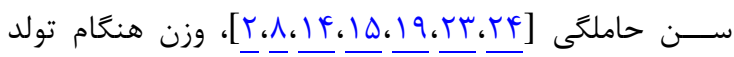

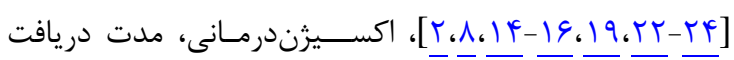

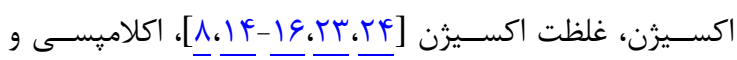

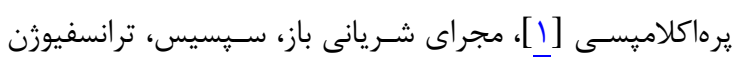

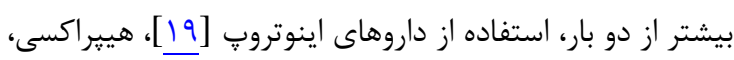

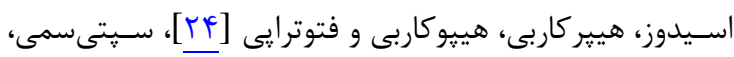

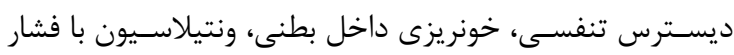

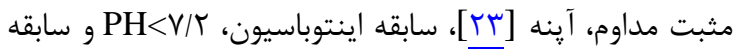
خونريزى داخل بطنى [1]] كزارش شده است.

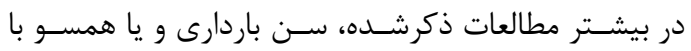

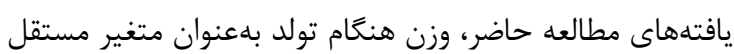
اثر گذار بر بروز رتينوياتى نارسى ذكر شده است. با توجه به اينكه ونه 
اســت كه نتايج اين مطالعه با منافع نويســندكان در تعارض نمى باشد.

\section{REFERENCES}

1. Yau GS, Lee JW, Tam VT, Liu CC, Yip S, Cheng E, et al. Incidence and risk factors of retinopathy of prematurity from 2 neonatal intensive care units in a Hong Kong Chinese population. Asia Pac J Ophthalmol. 2016;5(3):185-91. PMID: 27183289 DOI: 10.1097/APO.0000000000000167

2. Kola M, Haciö̈žLu DL, Erdol H, Turk A, Aslan Y. Determination of regional screening criteria for retinopathy of prematurity in the Eastern Black Sea region of Turkey. Turk J Med Sci. 2016;46(2):381-7. PMID: 27511500 DOI: 10.3906/sag-1410-97

3. Gibson DL, Sheps SB, Schechter MT, Wiggins S, McCormick AQ. Retinopathy of prematurity: a new epidemic? Pediatrics. 1989;83(4):486-92. PMID: 2927986

4. Augsburger JJ, Bornfeld N. Ophtalmology. St. Louis, MO: Mosby; 2004. P. 1097-102.

5. Mostafa Gharehbaghi M, Peirovifar A, Sadeghi K. Plasma leptin concentrations in preterm infants with retinopathy of prematurity (ROP). Iran J Neonatol. 2012;3(1):12-6. DOI: 10.22038/IJN.2012.275

6. Mustafa Gharebaghi M, Sadegh K, Zarghami N, Mostafizi H. Indices of vascular endothelial growth factor, leptin and insulin-like growth factor in retinopathy of prematurity. Urmia Univ Med J. 2012;23(2):91. [Persian]

7. Naderian G, Iranpour R, Mohammadizadeh M, Najafabadi FF, Badiei Z, Naseri F, et al. The frequency of retinopathy of prematurity in premature infants referred to an ophthalmology clinic in Isfahan. J Isfahan Med Sch. 2011;29(128):1-5. [Persian]

8. Nakhshab M, Ahmadzadeh Amiri A, Dargahi S, Farhadi R, Yazdani J. The incidence rate of retinopathy of prematurity and related risk factors: a study on premature neonates hospitalized in two hospitals in sari, Iran, 2014-2015. J Kerman Univ Med Sci. 2016;23(3):296-307. [Persian]

9. Mohammadi SF, Delshad H, Ghassemi F. Retinopathy of prematurity. $2^{\text {nd }}$ ed. Tehran: Aftab Publication; 2014. [Persian]

10. Edy Siswanto J, Sauer PJ. Retinopathy of prematurity in Indonesia: incidence and risk factors. $J$ Neonatal Perinat Med. 2017;10(1):85-90. PMID: 28304327 DOI: $10.3233 /$ NPM-915142

11. Gebesce A, Uslu H, Keles E, Yildirim A, Gurler B, Yazgan $\mathrm{H}$, et al. Retinopathy of prematurity: incidence, risk factors, and evaluation of screening criteria. Turk $J$ Med Sci. 2016;46(2):315-20. PMID: 27511491 DOI: 10.3906/sag1407-127

12. Senthil MP, Salowi MA, Bujang MA, Kueh A, Siew CM, Sumugam K, et al. Risk factors and prediction models for retinopathy of prematurity. Malays J Med Sci. 2015;22(5):5763. PMID: 28239269

13. Fajolu IB, Rotimi-Samuel A, Aribaba OT, Musa KO, Akinsola FB, Ezeaka VC, et al. Retinopathy of prematurity and associated factors in Lagos, Nigeria. Paediatr Int Child Health. 2015;35(4):324-8. PMID: 26744157 DOI: 10.1080/20469047.2015.1109277

14. Karkhaneh R, Mousavi SZ, Riazi-Esfahani M, Ebrahimzadeh SA, Roohipoor R, Kadivar M, et al. Incidence and risk factors of retinopathy of prematurity in a tertiary eye hospital in Tehran. Br J Ophthalmol. 2008;92(11):1446-9. PMID: 18728050 DOI: $10.1136 / \mathrm{bjo} .2008 .145136$

15. Fouladinejad M, Motahari MM, Gharib MH, Sheishari F, Soltani M. The prevalence, intensity and some risk factors of retinopathy of premature newborns in Taleghani Hospital, Gorgan, Iran. J Gorgan Univ Med Sci. 2009;11(2):51-4. [Persian]

16. Daraie G, Nooripoor S, Ashrafi AM, Ghorbani R. Incidence of retinopathy of prematurity and some related factors in premature infants born at Amir al- Momenin hospital in Semnan, Iran. Koomesh. 2016;17(2):297-303. [Persian]

17. Gerull R, Brauer V, Bassler D, Laubscher B, Pfister RE, Nelle

$$
\begin{aligned}
& \text { يزشــكى مصــوب دانشــاه علوم يزشــكى همدان مىباشـــد. }
\end{aligned}
$$

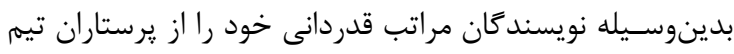

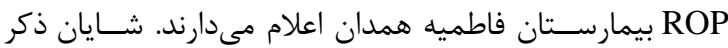

$\mathrm{M}$, et al. Incidence of retinopathy of prematurity (ROP) and ROP treatment in Switzerland 2006-2015: a population-based analysis. Arch Dis Childhood Fetal Neonatal Ed. 2018; 103(4):F337-42. PMID: 28916563 DOI: 10.1136/archdischild2017-313574

18. Hwang JH, Lee EH, Kim EA. Retinopathy of prematurity among very-low-birth-weight infants in Korea: incidence, treatment, and risk factors. J Korean Med Sci. 2015;30(Suppl 1):S88-94. PMID: 26566363 DOI: $10.3346 / \mathrm{jkms} .2015$. 30.S1.S88

19. Thomas K, Shah PS, Canning R, Harrison A, Lee SK, Dow KE. Retinopathy of prematurity: risk factors and variability in Canadian neonatal intensive care units. $J$ Neonatal Perinat Med. 2015;8(3):207-14. PMID: 26485554 DOI: 10.3233/NPM-15814128

20. Cerman E, Balci SY, Yenice OS, Kazokoglu H, Celiker H, Eraslan M. Screening for retinopathy of prematurity in a tertiary ophthalmology department in Turkey: incidence, outcomes, and risk factors. Ophthalmic Surg Lasers Imag Retina. 2014;45(6):550-5. PMID: 25423635 DOI: $10.3928 /$ 23258160-20141118-10

21. Chow PPC, Yip WWK, Ho M, Lok JYC, Lau HHW, Young AL. Trends in the incidence of retinopathy of prematurity over a 10-year period. Int Ophthalmol. 2019;39(4):903-9. PMID: 29907928 DOI: 10.1007/s10792-018-0896-0

22. Ahuja AA, Reddy YC, Adenuga OO, Kewlani D, Ravindran M, Ramakrishnan R. Risk factors for retinopathy of prematurity in a district in South India: a prospective cohort study. Oman J Ophthalmol. 2018;11(1):33-7. PMID: 29563692 DOI: $10.4103 /$ ojo.OJO $97 \_2016$

23. Azami M, Jaafari Z, Rahmati S, Farahani AD, Badfar G. Prevalence and risk factors of retinopathy of prematurity in Iran: a systematic review and meta-analysis. BMC Ophthalmol. 2018;18(1):83. PMID: 29606108 DOI: 10.1186/s12886-0180732-3

24. Khatami SF, Yousefi A, Bayat GF, Mamuri G. Retinopathy of prematurity among 1000-2000 gram birth weight newborn infants. Iran J Pediatr. 2008;18(2):137-42.

25. Wallace DK, Kylstra JA, Phillips SJ, Hall JG. Poor postnatal weight gain: a risk factor for severe retinopathy of prematurity. J AAPOS. 2000;4(6):343-7. PMID: 11124668 DOI: $10.1067 / \mathrm{mpa} .2000 .110342$

26. Wu C, Lofqvist C, Smith LEH, VanderVeen DK, Hellstrom A, Consortium W. Importance of early postnatal weight gain for normal retinal angiogenesis in very preterm infants: a multicenter study analyzing weight velocity deviations for the prediction of retinopathy of prematurity. Arch Ophthalmol. 2012;130(8):992-9. PMID: 22491391 DOI: 10.1001/archophthalmol.2012.243

27. Binenbaum G, Ying GS, Quinn GE, Huang J, Dreiseitl S, Antigua J, et al. The CHOP postnatal weight gain, birth weight, and gestational age retinopathy of prematurity risk model. Arch Ophthalmol. 2012;130(12):1560-5. PMID: 23229697 DOI: 10.1001/archophthalmol.2012.2524

28. Hellstrom A, Engstrom E, Hard AL, Albertsson-Wikland K, Carlsson Br, Niklasson A, et al. Postnatal serum insulin-like growth factor I deficiency is associated with retinopathy of prematurity and other complications of premature birth. Pediatrics. 2003;112(5):1016-20. PMID: 14595040

29. Stoltz Sjostrom E, Ãohlund I, Ahlsson F, Engstrom E, Fellman V, Hellstrom A, et al. Nutrient intakes independently affect growth in extremely preterm infants: results from a population-based study. Acta Paediatr. 2013;102(11):106774. PMID: 23855971 DOI: 10.1111/apa.12359

30. Dani C, Reali MF, Bertini G, Martelli E, Pezzati M, Rubaltelli FF. The role of blood transfusions and iron intake on retinopathy of prematurity. Early Hum Dev. 2001;62(1):5763. PMID: 11245995 